\title{
Racial, age, and community level maternal disparities at an academic health center
}

\author{
Candace N Holloway, Gordon Lee Gillespie,, Beth Ann Clayton \\ College of Nursing, University of Cincinnati, USA
}

Received: June 17, 2021

Accepted: September 16, $2021 \quad$ Online Published: October 28, 2021

DOI: $10.5430 /$ ijh.v7n2p42

URL: https://doi.org/10.5430/ijh.v7n2p42

\begin{abstract}
Objective: Racial and ethnic minority women experience more maternal deaths and comorbid illnesses than non-Hispanic White women. The purpose of the current study was to identify if maternal health disparities exist at an urban academic health center. Methods: A retrospective chart review was conducted of a systematic random sample of women who delivered a child in 2017. The study setting was an urban academic health center Level III neonatal intensive care unit serving a high percentage of racial minority patients. Data were analyzed using relative risks (RR) with $95 \%$ confidence intervals.

Results: Findings reflect an increased risk for maternal complications for minority and older aged women. Specifically, risk was higher for Black (RR: 3.818) and Hispanic/Latino (RR: 2.354) women compared to non-Hispanic White women for cesarean section and for older women (age 35 years or older) compared to younger women for cesarean section (RR: 2.671) and preeclampsia (RR: 3.422). While White, non-Hispanic women did not incur pre-eclampsia or hemorrhage with intervention, minority women did experience these maternal complications.

Conclusions: Maternal health inequities exist within this sample of women giving birth at an academic health center. Healthcare providers can conduct self-assessments to determine their implicit biases that may be contributing to health disparities.
\end{abstract}

Key Words: Healthcare disparities, Obstetrics, Maternal mortality, Severe maternal morbidity, African American

\section{INTRODUCTION}

Racial and ethnic disparities exist in maternal health outcomes, and these disparities systematically and negatively affect less advantaged groups. ${ }^{[1]}$ Racial and ethnic minority women experience more maternal deaths and comorbid illnesses than non-Hispanic White women. Specifically, Black women are 2 to 4 times more likely to die from pregnancyrelated causes and researchers suggest that $60 \%$ of pregnancyrelated deaths are preventable, highlighting inequities in health care access and quality of care factors that contribute to racial disparities in maternal mortality and severe morbidity. ${ }^{[2]}$ Black women have higher rates of postpartum hemorrhage, infection, and pregnancy-related cardiovascular con- ditions. ${ }^{[3-5]}$ In a study including more than 100,000 women, pregnancy-related racial disparities were documented in areas of labor induction and cesarean birth rates. ${ }^{[5]}$

The literature cites various causes of overall disparities, including adverse health behaviors like smoking or drug use, poor nutrition, inadequate health care, low socioeconomic status, poor living conditions, and stress, to name a few. ${ }^{[6]}$ The World Health Organization ${ }^{[7]}$ considers the major contributors to health disparities to be the conditions in which people are born, grow, live, work, and age. In the United States, ethnic minorities, particularly Black persons, are more likely to be in socially and economically vulnerable positions.

*Correspondence: Gordon Lee Gillespie; Email: gillesg1@ucmail.uc.edu; Address: College of Nursing, University of Cincinnati, USA. 
Families headed by unmarried women are most vulnerable to poverty and most likely to be among the working poor, and race continues to stratify single mothers' likelihood of living at or below the poverty line. ${ }^{[6]}$

Campbell et al., ${ }^{[8]}$ Tangel et al., ${ }^{[6]}$ and Hameed et al. ${ }^{[9]}$ examined insurance coverage and found an association with public insurance (Medicaid) or no insurance coverage and higher risk of maternal death or severe maternal morbidity compared with private insurance. More specifically, they found associations between public insurance or lack of insurance and higher risk of cardiovascular, respiratory, and severe sepsis-related death and in-hospital mortality. Among Black women, national and state-level studies consistently found higher pregnancy-related mortality risks, even when controlling for factors such as insurance coverage, marital status, and medical conditions. ${ }^{[6]}$ Moreover, researchers reported increased case-fatality, failure-to-rescue (death in the setting of severe morbidity), and preventable maternal deaths among Black women, suggesting management of severe morbidity may play a role in death disparities. ${ }^{[2]}$

Overall, the literature suggests significant racial disparities in maternal mortality and morbidity exist and need to be identified in an effort to be ameliorated. ${ }^{[10]}$ However, the magnitude of racial perinatal disparities within institutions is often not assessed, and for healthcare institutions seeking to establish a system of quality improvement to eliminate racial disparities, leaders can focus on local measurement opportunities to identify strategies to improve outcomes. ${ }^{[11,12]}$ Ultimately, national statistics and the literature warrant the completion of a needs assessment to investigate whether maternal health disparities exist as a precursor to eliminating pregnancy-related disparities.

\section{MethodS}

\subsection{Study design}

This study used a retrospective chart review design of maternal visits for women who delivered an infant in 2017. The university-affiliated Institutional Review Board (IRB) determined this study to be "Not Human Subjects Research." The university IRB provides ethical oversight for the setting where this study was conducted. The revised Standards for Quality Improvement Reporting Excellence (SQUIRE) guidelines were used for the conduct and reporting of this project.

\subsection{Setting}

The study was conducted at an academic Level I trauma center in the Midwest United States capable of providing total care for every aspect of injury from prevention through rehabilitation. The study site also houses a Level III Neonatal
Intensive Care Unit, which provides medical and surgical care for the most complex and critically ill pregnant women and fetuses throughout antepartum, intrapartum, and postpartum care. On average, there are over 2,500 births per year at the study site.

\subsection{Patients}

The inclusion criteria included delivering a child between 1 January 2017 through 31 December 2017 and available data for all of the variables collected with no explicit exclusion criteria where 1,620 charts met the eligibility criteria. Subjects were chosen from the electronic health record database using systematic random sampling where every tenth chart was reviewed, averaging 14 charts per month for one year. In total, 162 health records were selected for the retrospective chart review.

\subsection{Measurements}

Independent variables were the maternal demographic variables extracted from the chart: age, race, type of insurance, zip code, and delivery mode (vaginal, cesarean section). Race was categorized as White, non-Hispanic; Black, non-Hispanic; Hispanic/Latino; and other racial groups, nonHispanic. Insurance was categorized as private health insurance, Medicaid/Medicare, and no insurance (self-pay).

Dependent variables were maternal outcomes: preeclampsia, hemorrhage, and mortality due to complications related to the pregnancy or delivery. These variables were extracted by reviewing providers' notes, summaries, and intraoperative reports. Hemorrhage was defined as more than 500 $\mathrm{ml}$ blood loss after vaginal delivery or more than $1,000 \mathrm{ml}$ blood loss after a cesarean delivery; administration of carboprost, methylergometrine, or blood products; or diagnosis of postpartum hemorrhage within 24 hours after delivery.

Additional variables of interest were community characteristics obtained from the U.S. Census Bureau (http: //data.c ensus.gov). Variables were percent of zip code population who were renters and percent of zip code population who were female head of household (no husband present). These two variables in addition to health insurance served as socioeconomic status indicators or proxies for social determinants of health.

\subsection{Data analysis}

IBM SPSS Statistics 26 (Armonk, NY) was used to manage and analyze the data. Descriptive statistics were calculated to describe maternal demographic variables, maternal outcomes, and community characteristics. Crosstabs with Chi-square statistics were generated to compare descriptive statistics for race, age, female head of householder, and 
housing occupancy (by renters) by insurance and maternal complications. Relative risks with $95 \%$ confidence intervals were computed to identify disparities between maternal outcomes (i.e., complications) for age groups, racial categories, and community characteristics. A Cramer's V was calculated yielding effect size 0.298 . A post hoc power analysis using G*Power 3.1.9.2 was conducted based on effect size 0.298, alpha 0.05 , and sample size 162 yielded $96.7 \%$ power.

\section{RESUlts}

The sample was predominantly age $17-34$ years $(n=146$, $90.1 \%$ ) with mean age 27.4 years and median age 27 years (range 17-44 years). The sample was primarily White, nonHispanic $(\mathrm{n}=68,42 \%)$ or Black, non-Hispanic $(\mathrm{n}=57$, $35.2 \%)$. Nearly one third $(n=53,32.7 \%)$ of the sample had a cesarean section performed as the method of delivery. The majority of the sample lived in a community with a high percentage of females as the head of householder $(n=106$, $65.4 \%)$ or housing occupied by renters $(n=98,60.5 \%)$. See Table 1 for additional demographic characteristics of the study sample.

Health disparity based on insurance and maternal complications was compared by race (see Table 2). The distribution of subjects with private insurance compared to Medicaid/Medicare was approximately equal for non-Hispanic White women and non-Hispanic Black women but considerably lower when compared to Hispanic/Latino women and other racial groups. Non-Hispanic, Black women and Hispanic/Latino women had a disproportionate percentage of maternal complications compared to non-Hispanic, White women. Insurance and maternal complications were compared by age (see Table 3 ). There was a higher percentage of the 35 and older age group with private insurance $(\mathrm{n}=$ $11,68.8 \%$ ) and experienced more maternal complications (e.g., $75 \%$ had cesarean section) compared to the $17-34$ years old age group. Insurance and maternal complications were compared by female head of householder and housing occupancy by renters (see Table 4). Women living in zip codes with higher percentages of persons serving as the head of household (no husband present) or renting their residence had higher percentages of Medicaid/Medicare insurance and maternal complications.

Table 1. Demographic characteristics of the study sample $(\mathrm{N}=162)$

\begin{tabular}{ll}
\hline Characteristics & n (\%) \\
\hline Age & $146(90.1)$ \\
17-34 years & $16(9.9)$ \\
35 years or older & \\
Race & $68(42.0)$ \\
White, non-Hispanic & $57(35.2)$ \\
Black, non-Hispanic & $26(16.0)$ \\
Hispanic/Latino & $11(6.8)$ \\
Other racial groups, non-Hispanic & \\
Maternal complications & $53(32.7)$ \\
Cesarean section & $22(13.6)$ \\
Pre-eclampsia & $0(0.0)$ \\
Eclampsia & $1(0.6)$ \\
Hemorrhage, without intervention & $13(8.0)$ \\
Hemorrhage, with intervention & $4(2.5)$ \\
Mortality & \\
Insurance & $101(62.3)$ \\
Medicaid/Medicare & $61(37.7)$ \\
Private & \\
Community characteristic (based on zip code) & \\
Female householder & \\
No husband present, 7.8\%-14.1\% & \\
No husband present, 14.2\%-49.6\% & $106(65.4)$ \\
Housing occupied by renters & \\
10.3\%-34.6\% & $64(39.5)$ \\
34.7\%-80.3\% & $98(60.5)$ \\
\hline & \\
\hline
\end{tabular}

Table 2. Comparison of health equity by race

\begin{tabular}{|c|c|c|c|c|}
\hline \multirow[b]{2}{*}{ Characteristics } & \multicolumn{4}{|c|}{ Racial/Ethnic Group } \\
\hline & $\begin{array}{l}\text { White, non-Hispanic, } \\
\text { n (\%) }\end{array}$ & $\begin{array}{l}\text { Black, non-Hispanic, } \\
\text { n (\%) }\end{array}$ & $\begin{array}{l}\text { Hispanic/Latino, } \\
\text { n (\%) }\end{array}$ & $\begin{array}{l}\text { Other racial groups, } \\
\text { non-Hispanic, n (\%) }\end{array}$ \\
\hline \multicolumn{5}{|l|}{ Insurance *,\# } \\
\hline Private insurance & $34(50.0)$ & 25 (43.9) & $1(3.8)$ & $1(9.1)$ \\
\hline Medicaid or Medicare insurance & $34(50.0)$ & $32(56.1)$ & $25(96.2)$ & $10(90.9)$ \\
\hline \multicolumn{5}{|l|}{ Maternal complications \& } \\
\hline Cesarean section ${ }^{\#}$ & $10(14.7)$ & $32(56.1)$ & $9(34.6)$ & $2(18.2)$ \\
\hline Pre-eclampsia \# & $0(0.0)$ & $13(22.8)$ & $8(30.8)$ & $1(9.1)$ \\
\hline Hemorrhage, with intervention ${ }^{\dagger}$ & $0(0.0)$ & $10(17.5)$ & $1(3.8)$ & $2(18.2)$ \\
\hline Mortality & $1(1.5)$ & $2(3.5)$ & $1(3.8)$ & $0(0.0)$ \\
\hline
\end{tabular}

Note. ${ }^{*}$ Percentages compared by column; ${ }^{*} p<.001 ;{ }^{\&}$ Percentages compared by row; ${ }^{\dagger} p=.002$ 
Table 3. Comparison of health equity by age group

\begin{tabular}{lll}
\hline Characteristic & Age 17-34 years, n (\%) & Age 35 years or older, n (\%) \\
\hline Insurance * & & \\
$\quad$ Private insurance & $50(34.2)$ & $11(68.8)$ \\
Medicaid or Medicare insurance & $96(65.8)$ & $5(31.3)$ \\
Maternal complications & & \\
Cesarean section ${ }^{*}$ & $41(28.1)$ & $12(75.0)$ \\
Pre-eclampsia \& & $16(11.0)$ & $6(37.5)$ \\
Hemorrhage, with intervention & $11(7.5)$ & $2(12.5)$ \\
Mortality & $4(2.7)$ & $0(0.0)$ \\
\hline
\end{tabular}

Note. ${ }^{*} p=.007 ;{ }^{\#} p<.001 ;{ }^{\&} p=.003$

Table 4. Comparison of health equity by community characteristic

\begin{tabular}{|c|c|c|c|c|}
\hline \multirow{2}{*}{ Characteristic } & \multicolumn{2}{|c|}{ Female householder, no husband present, n (\%) } & \multicolumn{2}{|c|}{ Housing occupancy by renters, n (\%) } \\
\hline & Low percentage & High percentage & Low percentage & High percentage \\
\hline \multicolumn{5}{|l|}{ Insurance } \\
\hline Private insurance & $20(35.7)$ & $41(38.7)$ & $22(34.4)$ & 39 (39.8) \\
\hline Medicaid or Medicare insurance & $36(64.3)$ & $65(61.3)$ & $42(65.6)$ & $59(60.2)$ \\
\hline \multicolumn{5}{|l|}{ Maternal complications } \\
\hline Cesarean section * & $13(23.2)$ & $40(37.7)$ & $15(23.4)$ & $38(38.8)$ \\
\hline Pre-eclampsia ${ }^{\#}$ & $3(5.4)$ & 19 (17.9) & $5(7.8)$ & $17(17.3)$ \\
\hline Hemorrhage, with intervention & $5(8.9)$ & $8(7.5)$ & $6(9.4)$ & $7(7.1)$ \\
\hline Mortality & $2(3.6)$ & $2(1.9)$ & $2(3.1)$ & $2(2.0)$ \\
\hline
\end{tabular}

Note. ${ }^{*}$ Housing occupancy, $p=.042 ;{ }^{\#}$ Female householder, $p=.026$

Table 5. Comparison of relative risk for maternal complications by age, race, and community characteristics

\begin{tabular}{|c|c|c|c|c|}
\hline \multirow[b]{2}{*}{ Characteristics } & \multicolumn{4}{|c|}{ Maternal Complications, RR (95\% CI) } \\
\hline & Cesarean section & Pre-eclampsia & $\begin{array}{l}\text { Hemorrhage, with } \\
\text { intervention }\end{array}$ & Mortality \\
\hline \multicolumn{5}{|l|}{ Age } \\
\hline 17-34 years & 1.000 & 1.000 & 1.000 & Not able to calculate $^{*}$ \\
\hline 35 years or older & $2.671(1.819-3.921)$ & $3.422(1.563-7.491)$ & $1.659(0.403-6.833)$ & \\
\hline \multicolumn{5}{|l|}{ Race } \\
\hline White, non-Hispanic & 1.000 & & & 1.000 \\
\hline Black, non-Hispanic & $3.818(2.060-7.073)$ & Not able to calculate $\mathrm{e}^{\#}$ & Not able to calculate ${ }^{\#}$ & $2.386(0.222-25.639)$ \\
\hline Hispanic/Latino & $2.354(1.080-5.129)$ & & & $2.615(0.170-40.291)$ \\
\hline Other racial groups, non-Hispanic & $1.236(0.312-4.905)$ & & & Not able to calculate ${ }^{\&}$ \\
\hline \multicolumn{5}{|l|}{$\begin{array}{l}\text { Community characteristic (based } \\
\text { on zip code) }\end{array}$} \\
\hline \multicolumn{5}{|l|}{$\begin{array}{l}\text { Female householder, no husband } \\
\text { present }\end{array}$} \\
\hline Low percentage & 1.000 & 1.000 & 1.000 & 1.000 \\
\hline High percentage & $1.626(0.952-2.777)$ & $3.346(1.035-10.822)$ & $0.845(0.290-2.463)$ & $0.528(0.076-3.651)$ \\
\hline \multicolumn{5}{|l|}{ Housing occupied by renters } \\
\hline Low percentage & 1.000 & 1.000 & 1.000 & 1.000 \\
\hline High percentage & $1.654(0.996-2.749)$ & $2.220(0.862-5.719)$ & $0.762(0.268-2.164)$ & $0.653(0.094-4.520)$ \\
\hline
\end{tabular}


Lastly, we compared the relative risks for maternal complications and by age group, race, and community characteristics (see Table 5). Cesarean section as a maternal complication showed multiple health disparities. Women age 35 years or older had a $2.671(95 \%$ CI: 1.819, 3.921) times greater relative risk for having a cesarean section performed and 3.422 (95\% CI: 1.563, 7.491) times greater risk for experiencing pre-eclampsia compared to women age 17-34 years old. Non-Hispanic, Black women had a 3.818 (95\% CI: $2.060,7.073)$ times greater relative risk and Hispanic/Latino women had a 2.354 (95\% CI: 1.080, 5.129) times greater relative risk for cesarean section compared to non-Hispanic, White women. Women living in communities with a higher percentage of females as the head of householder (RR: 3.346, $95 \%$ CI: $1.035,10.822$ ) had higher relative risks for experiencing pre-eclampsia in relation to their comparison group. Health disparities also were found for pre-eclampsia and hemorrhage with intervention based on race, although risks could not be calculated due to no cases occurred in White, non-Hispanic women.

\section{Discussion}

Findings from this study provide information as to the problem of pregnancy-related morbidity and mortality disparity at a local urban health center. The relative risks reflect an increase in the likelihood of maternal complications for nonHispanic, Black and Hispanic/Latino women when compared to non-Hispanic, White women. Specifically, non-Hispanic, Black and Hispanic/Latino women when compared to nonHispanic, White women had greater relative risks for cesarean delivery (RR: 3.818, RR: 2.354, respectively). These findings are consistent with national statistics showing Black women are more likely to undergo a cesarean section, even for low-risk pregnancies, increasing their risk for severe morbidity and mortality. ${ }^{[1]}$ Our findings also are consistent with research showing Black women having higher rates of postpartum hemorrhage, puerperal infection, and venous thromboembolism. ${ }^{[3,5,13,14]}$ It is important to note that all racial groups, which were not non-Hispanic, White women, showed higher percentages for maternal complications reflecting the potential for an unconscious bias impacting the care of minority women. These findings reflect the need for an algorithm to help determine when parturient women should be transitioned from vaginal delivery to cesarean section delivery. Advanced practice nurses, including certified registered nurse anesthetists providing parturient anesthesia, can monitor women based on this algorithm and provide their expert recommendation for delaying or immediately transitioning to cesarean section delivery. In addition, advanced practice nurses can conduct case reviews of Black and Hispanic/Latino women at this facility to determine factors leading to the decision for cesarean section delivery and explore trends in racial disparities.

Efforts to resolve racial and ethnic disparities in maternal health are limited by lack of reliable data on patient identity including race and ethnicity, and patient and staff education on the importance for acquiring information related to identity. The limitations of available data on identity hamper the ability to understand and combat systemic disparities in care. Development of standardized data collection through creation of institutional dashboards may allow improved data collection and tracking to identify specific areas and causes surrounding maternal health disparities at varying health care institutions. Dashboards of measurements that not only illustrate the hospital systems overall results but stratify variables by race can aide in the quantification of disparities existing in local healthcare institutions. Optimally, data collected would include racial, demographic, and associated maternal health complications measured over time and allow for monitoring institutional performance and change. In addition, advanced practice nurses working in obstetrical settings can convene a community advisory board of women who previously delivered with and without poor maternal outcomes to discuss their parturient experiences. The women can be recruited from communities determined to have higher relative risks for poor maternal health outcomes. These experiences can inform the algorithm previously mentioned and address the societal and structural racism occurring within healthcare and disadvantaged communities. ${ }^{[15]}$

Comprehensive reviews have been performed to measure implicit bias in health care. A commonly used tool to assess implicit bias is the Implicit Association Test, which is available online. Hall et al. ${ }^{[16]}$ conducted a systematic review of 15 studies that assessed implicit bias in health care and found that the majority of studies focused on differences in treatment or outcomes for Black patients compared to White patients. The findings of the systematic review also indicated that adverse outcomes were most frequently related to patient-provider interactions, emphasizing the importance of communication and need for health care providers to be aware of their own implicit biases. The potential influence of implicit bias is especially relevant in the setting of environments prone to cognitive overload or high stress such as labor and delivery settings where reliance on automatic processes, when stereotypes and unconscious beliefs are more likely to be activated, are more frequently used. Implicit biases are malleable and remediable states that can be improved through increased personal awareness. Concerns about the effect of bias supports the recommendation to conduct implicit bias testing and education with all staff who participate 
in peripartum care.

Through personal awareness and overt acts to prevent implicit bias, healthcare providers can address the health disparities identified in the present study, particularly those experienced by non-Hispanic, Black and Hispanic/Latino women. Nurse educators and other obstetric leaders can serve as role models for addressing their personal implicit biases while bringing awareness to this problem by educating providers on the presence of these disparities, standardizing data collection with disparity dashboards, identifying possible causes of disparities (implicit and conscious), and implementing interventions to address the problem to ultimately improve maternal outcomes.

\section{Limitations}

This study was limited by a small sample size of 162 patient charts reviewed compared to the number of women on average giving birth at the study site. However, this limitation was mitigated by achieving greater than $95 \%$ power found during a post hoc power analysis. Also, the inability to control for adequacy of prenatal care, medication exposure, number of previous cesarean deliveries, Body Mass Index, and other clinical factors may be associated with the increased relative risks for severe maternal morbidity and mortality. Additional limitations include the results, while identifying associations, cannot be used to determine causation, the manualized process for data extraction could have yielded inaccuracies in the database used for analyses, and the electronic health record may have inaccurate data for total blood loss used to determine maternal hemorrhage.

\section{Conclusion}

Maternal health disparities exist within this sample of women giving birth at an academic health center. Disparities were predominantly identified for non-Hispanic, Black and Hispanic/Latino women compared to non-Hispanic, White women. Advanced practice nurses can review cases of poor maternal outcomes for trends. Healthcare providers can conduct self-assessments to determine their implicit biases that may be contributing to health inequities. By gaining awareness of maternal outcome trends and one's implicit biases, healthcare providers can begin to purposefully change their practices with the goal of improving maternal health outcomes. Future research is needed to determine if this proposed trending and personal awareness leads to desired improvements in maternal health outcomes.

\section{CONFlicts OF InTEREST Disclosure}

The authors declare no conflicts of interest.

\section{REFERENCES}

[1] Committee on Health Care for Underserved Women, American College of Obstetricians and Gynecologists. Committee opinion no. 649: Racial and ethnic disparities in obstetrics and gynecology. Obstet Gynecol. 2015; 126(6): e130-e134. PMid:26595584. https://doi.org/10.1097/A0G.0000000000001213

[2] Lawton B, MacDonald JE, Brown SA, et al. Preventability of severe acute maternal morbidity. Am J Obstet Gynecol. 2014; $210(6)$ : 557. PMid:24508582. https ://doi.org/10.1016/j.ajog.2013.12 .032

[3] Centers for Disease Control and Prevention. Pregnancy mortality surveillance system. 2020. Available from: https://www.cdc.go v/reproductivehealth/maternalinfanthealth/pmss.html

[4] Howland RE, Angley M, Won SH, et al. Determinants of severe maternal morbidity and its racial/ethnic disparities in New York City, 20082012. Maternal Child Health J. 2019; 23: 346-355. PMid:30712089. https ://doi.org/10.1007/s10995-018-2682-z

[5] Grobman WA, Bailit JL, Rice MM, et al. Racial and ethnic disparities in maternal morbidity and obstetric care. Obstet Gynecol. 2015; 125(6): 1460-1467. PMid:26000518. https ://doi.org/10.109 7/AOG.0000000000000735

[6] Tangel V, White RS, Nachamie AS, et al. Racial and ethnic disparities in maternal outcomes and the disadvantage of peripartum Black women: A multistate analysis, 2007-2014. Am J Perinatol. 2019; 36(8): 835-848. PMid:30396228. https ://doi.org/10.1 055/s-0038-1675207

Published by Sciedu Press
[7] World Health Organization. Maternal mortality. 2021. Available from: https://www.who.int/en/news-room/fact-sheets/ detail/maternal-mortality

[8] Campbell KH, Savitz D, Werner EF, et al. Maternal morbidity and risk of death at delivery hospitalization. Obstet Gynecol. 2013; 122(3): 627-633. PMid:23921870. https://doi.org/10.1097/A0G.0b $013 \mathrm{e} 3182 \mathrm{a} 06 \mathrm{f} 4 \mathrm{e}$

[9] Hameed AB, Lawton ES, McCain CL, et al. Pregnancy-related cardiovascular deaths in California: beyond peripartum cardiomyopathy. Am J Obstet Gynecol. 2015; 213(3): 379. PMid:25979616. https://doi.org/10.1016/j.ajog.2015.05.008

[10] Hirshberg A, Srinivas SK. Epidemiology of maternal morbidity and mortality. Semin Perinatol. 2017; 41(6): 332-337. PMid:28823579. https://doi.org/10.1053/j.semperi.2017.07.007

[11] Lange EMS, Rao S, Toledo P. Racial and ethnic disparities in obstetric anesthesia. Semin Perinatol. 2017; 41(5): 293-298. PMid:28600029. https://doi.org/10.1053/j.semperi.2017.04.006

[12] Toledo P, Sun J, Grobman WA, et al. Racial and ethnic disparities in neuraxial labor analgesia. Anesth Analg. 2012; 114(1): 172-178 https ://doi.org/10.1213/ANE.0b013e318239dc7c

[13] Creanga AA, Berg CJ, Syverson C, et al. Race, ethnicity, and nativity differentials in pregnancy-related mortality in the United States: 1993-2006. Obstet Gynecol. 2012; 120(2 Pt 1): 261-268. PMid:22825083. https://doi.org/10.1097/AOG.0b013e3182 $5 \mathrm{cb} 87 \mathrm{a}$

[14] Howell EA, Brown H, Brumley J, et al. Reduction of peripartum racial and ethnic disparities: A conceptual framework and maternal 
safety consensus bundle. Obstet Gynecol. 2018; 131(5): 770-782.

PMid:29683895. https://doi.org/10.1097/A0G.0000000000 002475

[15] Turienzo CF, Newburn M, Agyepong A, et al. Assessing inequities in maternal health among women living in communities of social disadvantage and ethnic diversity. BMC Public Health. 2021; 21:
176. PMid:33478445. https://doi.org/10.1186/s12889-021 $-10182-4$

[16] Hall JW, Chapman VM, Lee MK, et al. Implicit racial/ethnic bias among health care professionals and its influence on health care outcomes: A systematic review. Am J Public Health. 2015; 105(12): e60-e76. PMid:26469668. https://doi.org/10.2105/AJPH.2 015.302903 